\title{
Front Matter: Volume 11444
} Instrumentation 2020: Ultraviolet to Gamma Ray, 1144401 (7 January 2021); doi: $10.1117 / 12.2591710$ 


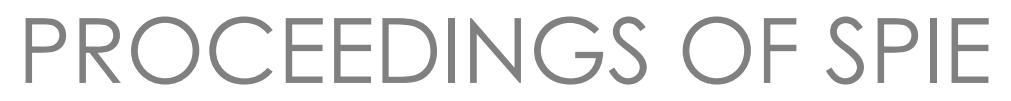

\section{Space Telescopes and Instrumentation 2020: Ultraviolet to Gamma Ray}

Jan-Willem A. den Herder

Shouleh Nikzad

Kazuhiro Nakazawa

Editors

14-18 December 2020

Online Only, California, United States

Sponsored and Published by

SPIE

Volume 11444

Part One of Two Parts 
The papers in this volume were part of the technical conference cited on the cover and title page. Papers were selected and subject to review by the editors and conference program committee. Some conference presentations may not be available for publication. Additional papers and presentation recordings may be available online in the SPIE Digital Library at SPIEDigitalLibrary.org.

The papers reflect the work and thoughts of the authors and are published herein as submitted. The publisher is not responsible for the validity of the information or for any outcomes resulting from reliance thereon.

Please use the following format to cite material from these proceedings:

Author(s), "Title of Paper," in Space Telescopes and Instrumentation 2020: Ultraviolet to Gamma Ray, edited by Jan-Willem A. den Herder, Shouleh Nikzad, Kazuhiro Nakazawa, Proceedings of SPIE Vol. 11444 (SPIE, Bellingham, WA, 2020) Seven-digit Article CID Number.

ISSN: 0277-786X

ISSN: 1996-756X (electronic)

ISBN: 9781510636750

ISBN: 9781510636767 (electronic)

Published by

SPIE

P.O. Box 10, Bellingham, Washington 98227-0010 USA

Telephone +1 3606763290 (Pacific Time) · Fax +1 3606471445

SPIE.org

Copyright (c) 2020, Society of Photo-Optical Instrumentation Engineers.

Copying of material in this book for internal or personal use, or for the internal or personal use of specific clients, beyond the fair use provisions granted by the U.S. Copyright Law is authorized by SPIE subject to payment of copying fees. The Transactional Reporting Service base fee for this volume is $\$ 21.00$ per article (or portion thereof), which should be paid directly to the Copyright Clearance Center (CCC), 222 Rosewood Drive, Danvers, MA 01923. Payment may also be made electronically through CCC Online at copyright.com. Other copying for republication, resale, advertising or promotion, or any form of systematic or multiple reproduction of any material in this book is prohibited except with permission in writing from the publisher. The CCC fee code is $0277-$ $786 \mathrm{X} / 20 / \$ 21.00$.

Printed in the United States of America by Curran Associates, Inc., under license from SPIE.

Publication of record for individual papers is online in the SPIE Digital Library.

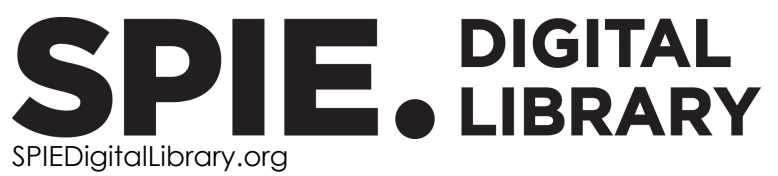

Paper Numbering: Proceedings of SPIE follow an e-First publication model. A unique citation identifier (CID) number is assigned to each article at the time of publication. Utilization of CIDs allows articles to be fully citable as soon as they are published online, and connects the same identifier to all online and print versions of the publication. SPIE uses a seven-digit CID article numbering system structured as follows:

- The first five digits correspond to the SPIE volume number.

- The last two digits indicate publication order within the volume using a Base 36 numbering system employing both numerals and letters. These two-number sets start with $00,01,02,03,04$, 05, 06, 07, 08, 09, OA, OB ... 0Z, followed by 10-1Z, 20-2Z, etc. The CID Number appears on each page of the manuscript. 


\section{Contents}

\section{Part One}

\section{ULTRAVIOLET I}

1144404 Ground calibration results of the JUICE ultraviolet spectrograph [1 1444-1]

1144405 EUV spectroscopy with the ESCAPE mission: exploring the stellar drivers of exoplanet habitability [11444-2]

1144406 Optical characterization of the Colorado Ultraviolet Transit Experiment (CUTE) CubeSat [11444-3]

$1144407 \quad$ Mapping diffuse emission in Lyman UV band [1 1444-4]

\section{ULTRAVIOLET II}

1144408 Progress report on the NASA probe mission concept, CETUS [1 1444-5]

1144409 INFUSE: a rocket-borne FUV integral field spectrograph [1 1444-6]

11444 OA SPARCS payload assembly, integration, and test update [11444-7]

\section{ULTRAVIOLET IV}

$1144401 \quad$ Development of high technology readiness level, all-reflective spatial heterodyne spectrometers for high resolving power/etendue remote sensing of extended emissions in the NUV to EUV [11444-15]

11444 OK Microchannel-plate detector development for ultraviolet missions [1 1444-17]

11444 OL UVSPEX/WSO-UV for Earth-like exoplanetary oxygen exospheres [11444-336]

\section{SOLAR}

11444 ON The Solar-C (EUVST) mission: the latest status [1 1444-19] 
ATHENA I

11444 OT Development status of the wide field imager instrument for Athena [1 1444-25]

\section{X-RAY OPTICS I}

$114441 \mathrm{C} \quad$ Sub-arcseconds to micro-arcsecond $\mathrm{x}$-ray imaging with Multi Image X-ray Interferometer Method (MIXIM): concept and scalabe mission plans [11444-44]

\section{X-RAY OPTICS II}

11444 1D Sub-arcseconds X-ray imaging with Multi-Image X-ray Interferometer Module (MIXIM): experimental results [11444-152]

$11444 \mathrm{IE} \quad$ An x-ray interferometry concept for the ESA Voyage 2050 programme [11444-153]

$1144416 \quad$ Advanced fabrication technologies for ultraprecise replicated mirrors for $\mathbf{x}$-ray telescopes [11444-155]

$11444 \mathrm{1H} \quad$ Towards volume manufacturing of high-performance soft $x$-ray critical-angle transmission gratings [11444-156]

SVOM

$114441 \mathrm{~K} \quad$ Calibration of a fully populated lobster eye optic for SVOM [11444-159]

SPEKTRUM RG

1144410 SRG/eROSITA in-flight background at L2 [11444-163]

SMALL/CUBE SATS I

$114441 R \quad$ The HERMES-technologic and scientific pathfinder [11444-166]

11444 is An innovative architecture for wide band transient monitor on board the HERMES nano-satellite constellation [11444-167]

$114441 \mathrm{~T}$ The scientific payload onboard the HERMES-TP and HERMES-SP CubeSat missions [11444-168]

iv 
11444 1V NinjaSat: an agile CubeSat approach for monitoring of bright $x$-ray compact objects [11444-170]

SMALL/CUBE SATS II

$114441 \mathrm{~B} \quad$ BurstCube: a CubeSat for gravitational wave counterparts [11444-172]

$114441 \mathrm{Z}$ The Mini Astrophysical MeV Background Observatory (MAMBO) CubeSat mission [1 1444-330]

XRISM

1144422 Status of X-ray Imaging and Spectroscopy Mission (XRISM) [1 1444-176]

1144423 Soft X-ray Imager (SXI) for Xtend onboard X-Ray Imaging and Spectroscopy Mission (XRISM) [1 1444-177]

1144425 On-ground calibration of XRISM/Xtend CCD [11444-179]

$1144426 \quad$ Planning in-flight calibration for XRISM [1 1444-180]

NEW

1144428 GEO-X (GEOspace X-ray imager) [11444-292]

11444 2A The Colibrì high-resolution x-ray telescope [11444-294]

$114442 C \quad$ The Arcus soft $x$-ray grating spectrometer explorer [11444-326]

EXTP

$114442 \mathrm{E} \quad$ Mission analysis and preliminary spacecraft design of the enhanced x-ray timing and polarimetry observatory [11444-298]

$1144421 \quad$ Investigating the effect of source contamination on eXTP/SFA [1 1444-199]

THESEUS

11444 2K The X/Gamma-ray Imaging Spectrometer (XGIS) on-board THESEUS: design, main characteristics, and concept of operation [11444-303] 
$114442 \mathrm{~L}$ The soft $\mathrm{x}$-ray imager on THESEUS: the transient high-energy survey and early universe surveyor [11444-304]

11444 2M The Infra-Red Telescope (IRT) onboard the THESEUS mission [1 1444-305]

\section{DETECTORS}

$1144420 \quad$ Identifying charged particle background events in x-ray imaging detectors with novel machine learning algorithms [11444-308]

$114442 Q \quad$ AstroPix: investigating the potential of silicon pixel sensors in the future of gamma-ray astrophysics [11444-310]

$114442 \mathrm{R} \quad$ A new spectroscopic imager for $\mathrm{x}$-rays from $0.5 \mathrm{keV}$ to $150 \mathrm{keV}$ combining a fully depleted pnCCD coupled to a columnar CsI(TI) scintillator with fano-noise-limited energy resolution and deep subpixel spatial resolution [1 1444-324]

HUBS

$114442 S$ HUBS: a dedicated hot circumgalactic medium explorer [11444-311]

$114442 \mathrm{~T} \quad$ Development of $\mathrm{x}$-ray focusing telescope for HUBS [11444-312]

$114442 \mathrm{U} \quad$ Preliminary design of a 4K mechanical cooling system for the HUBS mission [11444-313]

POLARIMETER

11444 2V POLAR-2: a large scale gamma-ray polarimeter for GRBs [1 1444-314]

$114442 Y \quad$ A small satellite version of a broad-band soft $x$-ray polarimeter [1 1444-317]

\section{GAMMA}

$114442 Z$ High-redshift gamma-ray burst for unraveling the Dark Ages Mission: Hiz-GUNDAM [1 1444-318]

1144430 A new tool for MeV astrophysics: the tunable Lave-lens [1 1444-319]

$1144431 \quad$ AMEGO: exploring the extreme multimessenger universe [11444-320]

1144434 Current status of the ComPair silicon tracker [11444-323] 
1144435 Results from the Advanced Scintillator Compton Telescope (ASCOT) balloon payload [11444-328]

POSTER SESSION: SVOM

1144438 Design and validation of onboard observation management software in satellite management unit of SVOM satellite [1 1444-268]

POSTER SESSION: SOLAR

11444 3J A sensitivity analysis of the updated optical design for EUVST on the Solar-C mission [11444-116]

11444 3K Thermal design of the Solar-C (EUVST) telescope [11444-117]

POSTERS SESSION: GENERAL

$114443 P \quad$ A comparison of trapped particle models in low Earth orbit [1 1444-74]

$114443 Q \quad$ Example telescope simulations with the AstroX telescope toolbox for McXtrace [11444-75]

POSTER SESSION: ATHENA

$114443 \mathrm{U} \quad$ Warm front end electronic modelization for the X-IFU ATHENA readout chain simulation [1 1444-45]

11444 3W Structural analysis of Athena WFI Large Detector Array [1 1444-47]

$114443 Y \quad$ Enhanced simulations on the Athena/WFI instrumental background [1 1444-49]

$1144441 \quad$ ATHENA warm ASIC for the X-IFU electronics [1 1444-52]

1144442 Reducing the Athena WFI charged particle background: results from Geant4 simulations [1 1444-53]

1144447 Technology development of Athena WFI frame processor electronics and verification of its real-time performance [11444-58]

11444 4A The cryogenic anticoincidence detector for ATHENA X-IFU: advancement in the project [11444-61] 


\section{PronttTwed Part Two}

\section{POSTER SESSION: ATHENA OPTICS}

$114444 G \quad$ Upgrade of the $x$-ray parallel beam facility XPBF 2.0 for characterization of silicon pore optics [11444-67]

11444 4J Qualification and performance of the Low-Energy X-ray Reflectometer (LEXR) [11444-70]

11444 4K Status of the Ir and Ir/SiC coating development for the Athena optics [11444-71]

11444 4L Design of a new long beam x-ray test facility for ATHENA [1 1444-72]

$114444 \mathrm{~N} \quad$ Balancing of residual stress in thin film iridium by utilizing chromium as an underlayer [11444-337]

POSTER SESSION: SPECTRUM RG

1144440 The eROSITA camera array on the SRG satellite [11444-260]

$114444 Q$ The calibration of eROSITA on SRG [11444-262]

$114444 S \quad$ SRG/eROSITA early phase and commissioning operations [1 1444-264]

POSTER SESSION: SMALL/CUBE SATS

$114444 U$ The HERMES-TP/SP background and response simulations [1 1444-248]

11444 4V GRBAlpha: a 1U CubeSat mission for validating timing-based gamma-ray burst localization [1 1444-249]

11444 4W MeVCube: a 6U CubeSat concept for MeV observations [1 1444-250]

$114444 \mathrm{X}$ Timing techniques applied to distributed modular high-energy astronomy: the HERMES project [11444-251]

1144450 The Payload Data Handling Unit (PDHU) on-board the HERMES-TP and HERMES-SP CubeSat Missions [1 1444-254]

1144454 Design, implementation, and validation of the electronics for the SPRITE CubeSat [11444-258]

1144456 A lunar CubeSat mission for high-sensitivity nuclear astrophysics [1 1444-332] 
POSTER SESSION: EINSTEIN

$1144457 \quad$ X-ray testing of the Einstein Probe follow-up x-ray telescope STM at MPE's PANTER facility [11444-193]

11444 5B Status of the follow-up x-ray telescope onboard the Einstein Probe satellite [11444-196]

POSTER SESSION: XRISM

11444 5C X-ray transmission measurements of the gate valve for the X-ray astronomy satellite XRISM [1 1444-285]

11444 5D The XRISM Science Data Center: optimizing the scientific return from a unique x-ray observatory [1 1444-286]

11444 5E Detail plans and preparations for the science operations of the XRISM mission [11444-287]

POSTERS SESSION: NEW

$114445 \mathrm{~K} \quad$ A detection algorithm for faint sources based on 1-d projection for a lobster-eye $\mathrm{x}$-ray imaging system [11444-227]

$114445 \mathrm{~L} \quad$ Development of a fast readout system of a CMOS image sensor for the time-domain astronomy [1 1444-228]

1144450 Super DIOS mission for exploring "dark baryon" [11444-231]

POSTER SESSION: POLARIMETRY

$114445 \mathrm{~V} \quad$ Concept of a CubeSat-based hard x-ray imaging polarimeter: cipher [1 1444-238]

$114445 X \quad$ The back-end electronics for the Imaging X-ray Polarimetry Explorer telescope [1 1444-240]

$114445 Y \quad$ Development and performance verification of a TPC polarimeter for high energy $\mathbf{x}$-rays [11444-241]

$114445 Z$ CAT grating alignment and testing for soft x-ray polarimetry [1 1444-242]

$1144460 \quad$ Ray-tracing a small orbital mission for soft-x-ray polarimetry [1 1444-243]

1144462 The Imaging X-ray Polarimetry Explorer (IXPE): technical overview III [1 1444-245] 
POSTER SESSION: GAMMA

1144467 SMILE-3: sky survey in MeV gamma-ray using the electron-tracking Compton telescope loaded on balloons [11444-211]

$114446 \mathrm{~A} \quad$ Trigger system for the ComPair instrument [11444-214]

11444 6D Development of a Compton telescope based on single-crystal diamond detectors and fast scintillators [11444-327]

11444 6E GALI: a gamma-ray burst localizing instrument [1 1444-329]

POSTER SESSION: ULTRAVIOLET

114446 Precision requirements for the POLLUX-LUVOIR spectropolarimeter [11444-123]

1144461 The detector for the far ultraviolet channel of the imaging instrument on board the Spektr-UF (WSO-UV) space telescope [11444-125]

$114446 Q \quad$ Miniaturized UV imager for heliophysics science investigations [1 1444-133]

11444 6V GLUV Pathfinder: setting up for rapid cadence UV monitoring of the transient universe [1 1444-138]

11444 6W Optical design of the Chromospheric LAyer Spectro-Polarimeter (CLASP2) [1 1444-139]

$114446 Y \quad$ Far-UV multi-object spectroscopy with digital micromirror devices (DMDs) [1 1444-141]

1144473 The World Space Observatory: ultraviolet mission: science program and status report [11444-146]

1144474 Field camera unit of the WSO-UV mission [1 1444-147]

1144476 Spectroscopic Investigation Of Nebular Gas (SING): a dedicated NUV spectrograph to study extended objects from a stable space platform [1 1444-149]

$1144478 \quad$ Mirrors for improved FUV observations [11444-151]

11444 7A Qualitative and quantitative test of digital micromirror device for next generation UV multiobject spectroscopy [11444-338]

POSTER SESSION: OPTICS

$114447 \mathrm{C} \quad \mathrm{X}$-ray performance and simulation study of lobster eye optics [1 1444-80] 
11444 7E Source position determination method of multiplexing lobster-eye optics [1 1444-82]

1144476 Development of a powerful x-ray generator with a small spot-size option at ISAS x-ray beamline facility [1 1444-84]

11444 7P New method to make a smooth surface on Carbon Fiber Reinforced Plastic (CFRP) substrate [1 1444-93]

$114447 R \quad$ Testing of the WXT optics at the University of Leicester [1 1444-95]

11444 7V VTXO: the virtual telescope for $x$-ray observations [1 1444-99]

$114447 \mathrm{~W}$ The development of a testbed for the x-ray interferometer mission [1 1444-100]

$1144488 \quad$ Lynx grating spectrometer design: optimizing chirped transmission gratings [1 1444-112]

114448 A Alignment of the Marshall Grazing Incidence X-ray Spectrometer (MaGIXS) telescope mirror and spectrometer optics assemblies [11444-331]

$114448 \mathrm{~B} \quad$ The 2nd generation of the $\mathrm{x}$-ray multi-foil optical system for rocket experiment [11444-339]

POSTER SESSION: EXTP

11444 8E Optical thermal filters for eXTP: manufacturing and characterization [1 1444-200]

$114448 \mathrm{~F} \quad$ Mechanical design and analysis of the eXTP satellite [1 1444-201]

POSTER SESSION: THESEUS

114448 P The XGIS instrument on-board THESEUS: Monte Carlo simulations for response, background, and sensitivity [1 1444-275]

$114448 Q \quad$ Scientific simulations and optimization of the XGIS instrument on board THESEUS [1 1444-276]

$114448 R \quad$ The XGIS instrument on-board THESEUS: the detection plane and on-board electronics [1 1444-277]

$114448 S$ The XGIS imaging system onboard the THESEUS mission [1 1444-278]

$114448 X \quad$ The development of the THESEUS SXI optics [1 1444-283]

$114448 Y$ Development of an imaging system for the THESEUS SXI instrument [1 1444-284] 
1144492 A software toolkit to simulate activation background for high energy detectors onboard satellites [11444-184]

1144493 A summary on an investigation of GAGG:Ce afterglow emission in the context of future space applications within the HERMES nanosatellite mission [1 1444-185]

1144494 Progress toward fast, low-noise, low-power CCDs for Lynx and other high-energy astrophysics missions [11444-186]

1144495 Radiation testing of a small pixel, CMOS compatible CCD [1 1444-187]

1144497 A revised model of the temporal behavior of the ACIS contamination layer on the Chandra X-ray Observatory [11444-190]

11444 9A Preliminary results of the pixel characterization for the Crystal Eye, a new $\mathbf{x}$ - and $\mathbf{y}$-ray satellite detector for multi-messenger astronomy [11444-325]

11444 9B MCPSim-Py: an open source python-based simulator of the performance of MCP photoncounting detectors [11444-333]

POSTER SESSION: HUBS

$114449 \mathrm{C}$ Development of TES-based x-ray microcalorimeters for HUBS [1 1444-217]

11444 9F Making mock HUBS observations with IllustrisTNG [1 1444-220]

1144496 Development of adiabatic demagnetization refrigerator for HUBS [1 1444-221]

$1144491 \quad$ Preliminary architecture of integrated cooling system for the HUBS mission [1 1444-223]

$114449 \mathrm{~J} \quad$ Developing x-ray microcalorimeters based on TiAu TES for HUBS [1 1444-224] 